\title{
A new enhanced recovery after surgery pathway for left-sided pancreatic cancer patients after distal pancreatectomy
}

\author{
Yue Wang", Jingfeng Li", Yuanchi Weng, Xiaxing Deng, Jiabin Jin, Chenghong Peng \\ Department of General Surgery, Ruijin Hospital Affiliated to Shanghai Jiao Tong University School of Medicine, Shanghai 200025, China \\ Contributions: (I) Conception and design: J Jin, C Peng; (II) Administrative support: C Peng; (III) Provision of study materials or patients: Y Weng, \\ X Deng; (IV) Collection and assembly of data: Y Wang, J Li; (V) Data analysis and interpretation: Y Wang, J Li; (VI) Manuscript writing: All authors; \\ (VII) Final approval of manuscript: All authors. \\ "These authors contributed equally to this work. \\ Correspondence to: Chenghong Peng; Jiabin Jin. Department of General Surgery, Ruijin Hospital Affiliated to Shanghai Jiao Tong University School of \\ Medicine, 197 RuiJin 2 Road, Shanghai 200025, China. Email: chhpeng@yeah.net; jjb11501@rjh.com.cn.
}

\begin{abstract}
Background: Pancreatic surgeries are one of the most complex surgical procedures in general surgery that require highly experienced surgical technics and intensive postoperative care. Meanwhile the application of enhanced recovery after surgery (ERAS) program in pancreatic surgery is limited. The aim of this study was to attempt a new ERAS pathway specifically for patients after distal pancreatectomy (DP).

Methods: Between May 2016 and November 2016, 29 patients undergoing DP in Shanghai Ruijin Hospital pancreatic disease center received the ERAS pathway. Meanwhile 19 patients were implemented traditional care. Complications, readmissions, mortality, length of stay (LOS) and drainage-tube-off day were compared and evaluated in the two groups.

Results: Median length of stay was significantly reduced (18.37 \pm 2.55 days in ERAS group; $27.42 \pm 7.60$ days in the control group) and drainage-tube-off days (5.89 \pm 2.41 versus $26.68 \pm 7.35$ days). And no patient discharged with a drainage tube in ERAS group compared while 7 patients $(36.8 \%)$ discharged with drainage tube in the conventional group. Postoperative pancreatic fistula (POPF) rate was decreased in ERAS group but not significantly. Readmission rates, as well as mortality, did not change over time. 9 patients were found postoperative abdominal fluid collections in ERAS group, and only 2 patients were confirmed real pancreatic fistula.

Conclusions: Implementing the new ERAS program for DP may contribute to a decrease of LOS and drainage-tube-off days with a promising outcome. Therefore, an ERAS pathway specifically for DP should be established.
\end{abstract}

Keywords: Postoperative recovery; distal pancreatectomy; pancreatic surgery; enhanced recovery after surgery (ERAS)

Submitted Jun 01, 2019. Accepted for publication Oct 10, 2019.

doi: $10.21037 /$ tcr.2019.10.26

View this article at: http://dx.doi.org/10.21037/tcr.2019.10.26

\section{Introduction}

Pancreatic surgery is highly technically challenging surgical procedure in general surgery field. With the development of surgical technics and medical instruments, patients undergoing pancreatic surgeries in specialized centers and received advanced perioperative care have resulted in less than $5 \%$ mortality rate (1). However, morbidity such as postoperative pancreatic hemorrhage $(\mathrm{PPH})$ and postoperative pancreatic fistula (POPF) rates still remain as high as $3-45 \%(2-4)$. To further boost the shortterm outcome, the Enhanced Recovery After Surgery (ERAS) concept has been created. The ERAS program was firstly attempted by Kehlet in the early 1990s for gastrointestinal surgery (5). In pancreatic surgery, several 
centers came up with different ERAS pathways that all could improve perioperative outcome and especially reduce morbidity of surgery (6-9). In 2012, the ERAS pancreas collaborative group set out a consensus ERAS protocol for pancreaticoduodenectomy (PD) (10). However, there has been no ERAS program designed specifically for distal pancreatectomy (DP). Considering the difference of both surgeries about anatomical features and operation ways, it is necessary to create an entirely new ERAS pathway for DP surgery. The aim of the study was to evaluate the effects of implementing the new ERAS pathway for DP.

\section{Methods}

\section{Patients and methods}

All 29 patients undergoing DP received the new ERAS pathway in Shanghai Ruijin Hospital between May 2016 and November 2016 were included. These patients were compared with a group of patients after surgeries in the same period treated with conventional way. We adapted the ERAS guidelines for pancreaticoduodenectomy in 2012 (10) and our own ERAS protocol for PD (11) to develop this new ERAS program and applied it to patients in ERAS group as shown in Table 1.

The standard of removing drainage tube was created by several references and combined with our own experience, and effective (11-14). The same surgical team carried out all surgeries on the ERAS group and conventional group. All patients were enrolled in a database retrospectively. The following data were extracted from database: age, sex, BMI, comorbidity and routine liver function test including bilirubin level. Operative variables included type of surgery, duration of surgery, blood loss and blood transfusions. Postoperative outcomes were the length of stay (LOS), complications, mortality, and readmission rate. Unplanned reoperation, mortality, readmission, and complications were recorded before discharge or within 30 days after discharge. POPF, PPH and delayed gastric emptying (DGE) were defined according to the International Study Group of Pancreatic Surgery $(2,4,15)$. When patients had symptoms such as leukocytosis, abdominal pain, and fever associated with abdominal effusion, a CT-guided insertion of a new percutaneous drainage (PCD) catheter would be performed. The drainage tube was removed after evaluation of CT scan. After discharge, a follow-up abdominal CT scan was performed every 3 months after surgery.

This study was approved by the ethics committee of
Ruijin Hospital. And the study protocol was also approved by the institutional review board at Ruijin Hospital.

\section{Statistical analysis}

Statistical analysis was performed using statistical package for the social sciences (SPSS) software version 17.0 (SPSS Inc, Chicago, III, USA). Fishers exact test or Student's $t$-test was used to evaluate significant differences. Descriptive data are reported as mean $\pm \mathrm{SD}$ and median (range), or number of patients and percentage. $\mathrm{P}$ value $<0.05$ was considered statistically significant.

\section{Results}

\section{Patients, preoperative variables and operative variables}

Demographic patient data, preoperative variables, and intra-operative variables are shown in Table 2. There was no significant difference in the baseline parameters of patients between the ERAS and conventional groups. Operative variables of these two groups also did not significantly differ.

\section{Postoperative parameters of the 2 groups}

The time of nasogastric tube (NGT) removed days, drainage-tube-off days and postoperative length of stay were remarkably reduced in the ERAS group (Table 3). Although this study required two weeks fasting of patients, exhaust defecation time shows no difference between the two groups. Difference was observed in start with oral soft solid food between two groups because the ERAS group started intake until postoperative day (POD) 14 days. What we should emphasize is this: In ERAS group, there was no patient discharged with abdominal drainage tube. Nevertheless, there were still 7 (36.8\%) patients treated with conventional procedure discharged with drainage tube.

\section{Postoperative complications and mortalities of the 2 groups}

The differences of postoperative complications and mortalities in detail between two groups are shown in Table 4. In ERAS group, after early removal of the abdominal drainage tube, Abdominal effusion was present in nine patients. Three of those patients due to symptomatic encapsulated effusion received CT-guided puncture 
Table 1 Protocols for the ERAS program and traditional care

\begin{tabular}{|c|c|c|}
\hline Hospital stay & ERAS group & No-ERAS group \\
\hline \multirow[t]{3}{*}{ Day before surgery } & Normal oral nutrition until $10 \mathrm{PM}$ & Overnight fasting \\
\hline & No pre-anaesthetic medication & Oral bowel preparation \\
\hline & No bowel preparation & \\
\hline \multirow[t]{5}{*}{ Day of surgery } & Fasting for liquids 6 hours before surgery & Intravenous fluids \\
\hline & $\begin{array}{l}\text { Elastomeric analgesia pump:(flurbiprofen } 300 \mathrm{mg} \text {, } \\
\text { tramadol } 60 \mathrm{mg} \text { in } 100 \mathrm{~mL} \text { saline solution) }\end{array}$ & Antimicrobial prophylaxis \\
\hline & Avoidance of excessive i.v. fluid & No intravenous analgesia \\
\hline & Antimicrobial prophylaxis & $\begin{array}{l}\text { Octreotide (Sandostain) } 100 \mu \mathrm{g} \text { q8h subcutaneous } \\
\text { injection }\end{array}$ \\
\hline & $\begin{array}{l}\text { Octreotide (Sandostatin) } 100 \mu \mathrm{g} \text { q8h subcutaneous } \\
\text { injection }\end{array}$ & \\
\hline \multirow[t]{6}{*}{ Day1 } & Patient sent back to surgical ward & Total parenteral nutrition (TPN) \\
\hline & Removal of NGT if $<200 \mathrm{~mL}$ & \\
\hline & Urinary catheters removed & \\
\hline & Continue mobilization 4 times per day & \\
\hline & Sip of warm water at rate $\leq 30 \mathrm{~mL} / \mathrm{L}$ & \\
\hline & Total parenteral nutrition (TPN) & \\
\hline Day5 & - & Removal of NGT if $<100 \mathrm{~mL}$ with flatus or defecation \\
\hline \multirow[t]{2}{*}{ Day7 } & - & Soft solid diet \\
\hline & & $\begin{array}{l}\text { Removal of drainage tube if } A M Y<300 \mathrm{U} / \mathrm{L} \text { and } \\
\text { volume }=0 \mathrm{~mL} \text { in two days }\end{array}$ \\
\hline \multirow[t]{3}{*}{ Day14 } & Soft solid diet & - \\
\hline & Stop TPN support & \\
\hline & Stop octreotide (Sandostatin) & \\
\hline \multirow[t]{6}{*}{ Discharge criteria } & Absence of fever for $>48 \mathrm{~h}$ & Absence of fever for $>48 \mathrm{~h}$ \\
\hline & Able to take solid food & Able to take solid food \\
\hline & Passage of normal stools & Passage of normal stools \\
\hline & Adequate mobilization & Adequate mobilization \\
\hline & No drainage tube & With/without drainage tube \\
\hline & Acceptance of discharge by the patient & Acceptance of discharge by the patient \\
\hline
\end{tabular}


Table 2 Demographic and intra-operative parameters of the 2 groups

\begin{tabular}{lccc}
\hline Parameters & ERAS $(\mathrm{n}=29)$ & Conventional $(\mathrm{n}=19)$ & $\mathrm{P}$ value \\
\hline Age & $54.41 \pm 15.21[22-81]$ & $61.78 \pm 13.18[44-94]$ & 0.201 \\
Sex (M/F) & $14 / 15$ & $7 / 12$ & 0.555 \\
BMI & $21.93 \pm 2.99(17-26.56)$ & $21.62 \pm 2.99(16.83-23.67)$ & 0.657 \\
Diabetes & $4(13.79 \%)$ & $2(10.52 \%)$ & 1 \\
Cardiac vascular disease & $3(10.34 \%)$ & 0 & 0.267 \\
Preoperative total bilirubin (mmol/L) & $15.65 \pm 5.31(7.8-30.5)$ & $13.39 \pm 6.34(3.6-27.7)$ & 0.125 \\
Preoperative albumin (g/L) & $37.38 \pm 5.69[21-46]$ & $39.58 \pm 6.19[25-53]$ & 0.291 \\
Operation & $143.3 \pm 54.91[45-300]$ & $171.1 \pm 63.59[50-300]$ & 0.101 \\
Operating time (time) & $236.4 \pm 307[20-1,500]$ & $215 \pm 147.7[20-500]$ & 0.450 \\
Blood loss (mL) & $3(10.34 \%)$ & $6(31.57 \%)$ & 0.127 \\
Blood transfusion rate & $21(72.41 \%)$ & $19(100 \%)$ & 0.015 \\
With splenectomy & &
\end{tabular}

Table 3 Postoperative parameters of the 2 groups

\begin{tabular}{lccc}
\hline Parameters & ERAS $(\mathrm{n}=29)$ & Conventional $(\mathrm{n}=19)$ & $\mathrm{P}$ value \\
\hline Nasogastric tube removed (days) & 1 & $3.94 \pm 1.74[1-7]$ & $<.0001$ \\
Oral soft solid diet (days) & 14 & $13.68 \pm 8.25[6-33]$ & 0.004 \\
Exhaust defecation time & $2.86 \pm 1.50[1-7]$ & $4.36 \pm 4.43[2-21]$ & 0.134 \\
Drain tube removed (days) & $5.89 \pm 2.41[3-14]$ & $26.68 \pm 7.35[16-46]$ & $<0.001$ \\
Postoperative hospital stay (days) & $18.37 \pm 2.55[15-25]$ & $27.42 \pm 7.60[16-47]$ & $<0.001$ \\
Discharge with drainage & 0 & $7(36.83 \%)$ & 0.0007 \\
\hline
\end{tabular}

Table 4 Postoperative complications and mortalities of the 2 groups

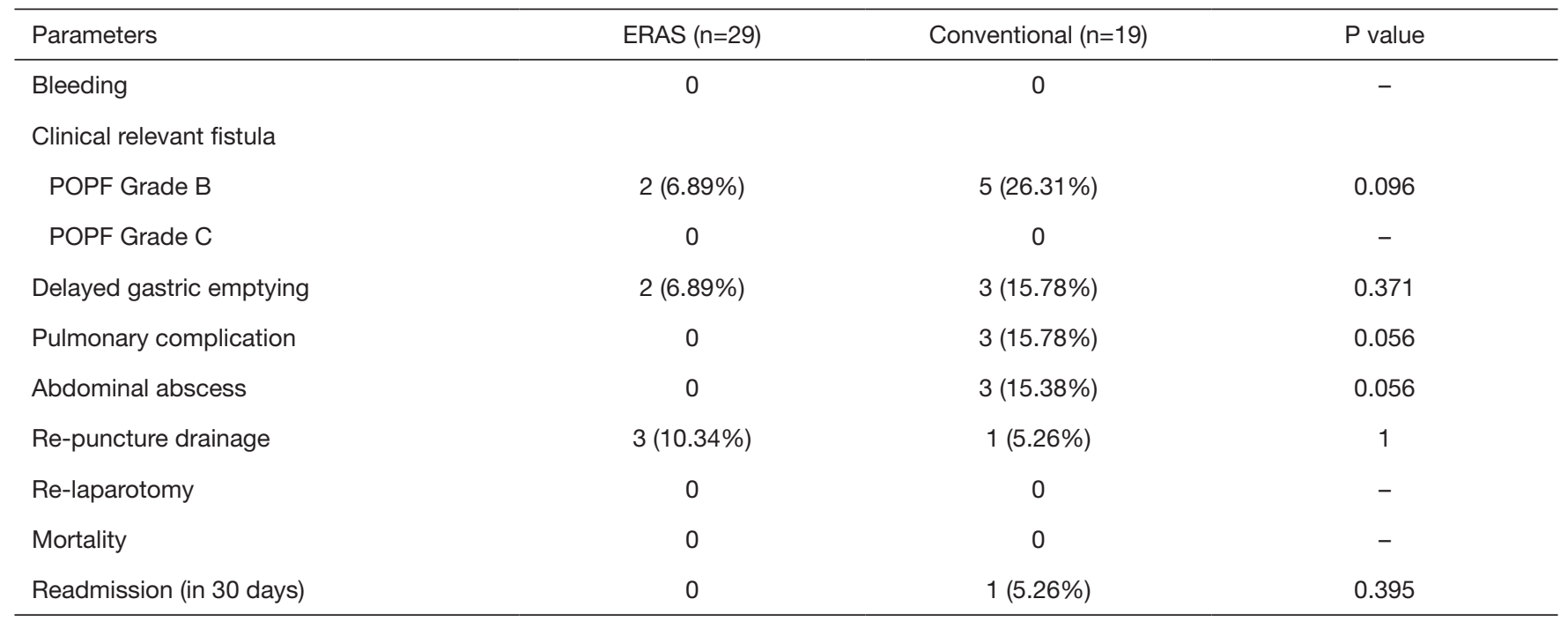



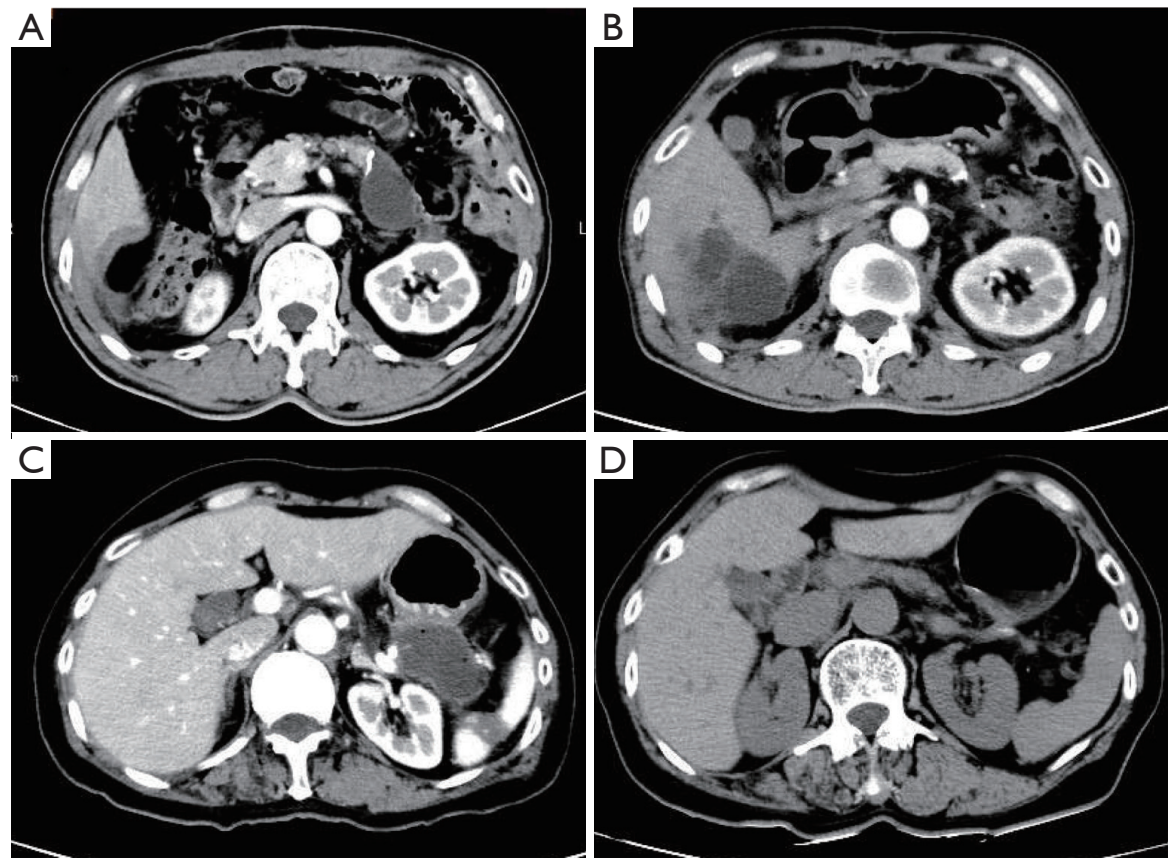

Figure 1 Postoperative CT findings after DP (A,C). Postoperative fluid collection is initially dispersed around the pancreatic stump and then became a round-shaped pseudocyst (B,D). Fluid collection was observed spontaneous regression.

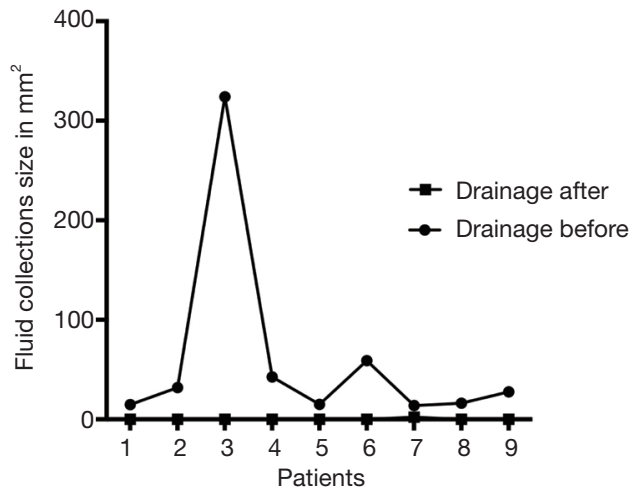

Figure 2 Course of 9 fluid collections. Size of fluid collections in $\mathrm{mm}^{2}$ changing after follow-up (2-3 CT examinations).

drainage. With amylase assay confirmed, 2 of them were diagnosed as real pancreatic fistula. While the other six patients also presenting encapsulated effusion spontaneously absorbed (Figure 1). The course of all nine patients' fluid collections was shown in Figure 2.

\section{Discussion}

Distal pancreatectomy (DP) is performed on patients with a benign and malignant tumor of the distal pancreas, which has been a mature operation. After nearly 20 years development, surgical technique and perioperative management have remarkably reduced the rates of operative morbidity and mortality after DP (16-18). However, the overall incidence of POPF in patients undergoing DP is as high as $12-31 \%(16-18)$. In addition, POPF is not a life-threatening condition in most cases, but once associated with additional complications such as intraabdominal abscess or hemorrhage will lead to a prolonged hospital stay or even death. Sauvanet suggested that POPF originating from pancreaticoduodenectomy (PD) seems to have a worse prognosis than POPF from a pancreatic remnant (DP) (19). This may be due to the activation of pancreatic juice. Surgical procedures may contribute to the differences between pancreatic fistulas that require pancreaticojejunostomy (such as PD and central pancreatic resection) and those not (DP and enucleation). Pratt suggested that clinically relevant fistulas after PD require more aggressive management (20). On the other hand, while POPF that occur after distal resections often require conservative treatments. If fluid collections (caused by pancreatic fistula or not) do not spontaneously absorb then CT-guided percutaneous drainage will be arranged. 
Several decades, all the efforts that pancreatic surgeons did were to decrease POPF rate to avoid significant clinical complications and high costs. In our study, our new ERAS pathway leads to clinical POPF rate as low as $6.89 \%$ compared with $26.31 \%$ in conventional group.

Along with putting forward the rapid recovery concept, the ERAS strategy has been practiced in plenty of major surgical centers, which advocates early open diet, intraoperative control rehydration, early postoperative analgesia, and activities. Early removal of drainage tube especially has an influence on the traditional concept of drainage tube processing strategy. While open early diet, inevitably stimulating secretion of the pancreas, early removal of the tube may not fully drain effusion area. Especially after resection of the pancreatic body because of the existence of the sphincter of Oddi, the pressure of main pancreatic duct pressure is higher than the pancreas residual surface. The reason for this might be why patients after DP have a higher incidence of postoperative pancreatic fistula than those after PD. And the treatment of acute pancreatitis experience tells us to keep the pancreas inhibition can significantly reduce the effusion around the pancreas. So how to unify early oral food intake and early removal of drainage, we need to further discuss. That is why we are trying to set ERAS strategy specifically for distal pancreatectomy. Fluid collections (FCs) after DP occur frequently, and several studies revealed that FCs after DP are frequent and harmless findings, only a few patients require therapeutic interventions (21-24). And to reduce the FCs around the pancreas for pancreatitis patients, fasting is one of the most important conservative treatments (23). In this study, we let the patient fasting for 14 days (the encapsulated effusion formation generally needs 14 days) meanwhile giving them total parenteral nutrition (TPN) for nutrition support at the same time. Before discharging, CT examination showed that nine (31\%) patients experienced postoperative peritoneal effusion. Compared with a study about DP in Germany (22), fasting, one of key methods in our ERAS program, may reduce pancreatic secretion by food stimulating and lead to lower incidence of postoperative abdominal fluid collections ( $31 \%$ versus $40.25 \%)$.

In the past, treatment conditions were limited, surgeons hoped that through the drainage in treating of pancreatic fistula, now which can be achieved by other means such as somatostatin, regular application of enteral nutrition and so on. Somatostatin and application of TPN can make the pancreas in rest status, and then reduce pancreatic juice secretion, which naturally reduces the occurrence of pancreatic fistula.

Besides the uses of somatostatin or its analogs in highrisk patients (25), the occurrence of POPF and FCs can decrease sharply. In the present, the drainage tube is no less that important. And the existence of the drainage tube may lead to retrograde infection of abdominal cavity. Therefore, we recommend early removal of drainage tube. Our paper emphasized the removal of the drainage in the hospital which was the advantage of ERAS protocol, and maintaining fasting within 2 weeks might contribute it. Different national policy in china made it impossible to discharge most of the patients with the drainage as we don't have a community or home care for these patients who received a recent pancreatectomy and with abdominal drainage.

Pancreatic surgeons around the world inevitably all face the same problem: there is a shortage of medical resources, especially the specialized pancreatic centers or specialist surgeons. And for better use of medical resource and earlier discharge most hospitals often require patients discharged with a drainage tube. Thus in several clinical studies, hospitalization days compared with other discharge with drainage tube hospitals had decreased dramatically. Often patient discharged with drainage has clinical pancreatic fistula, fluid amylase can be hundreds of thousands and volume can be from dozens to hundreds of milliliters. Although the treatment of pancreatic fistula only needs patience and unobstructed drainage, for such patients, to some extent had some similarities with those patients in need of interventional drainage. If we discharge those patients with Grade B POPF who received interventional drainage, whether patients' security is feasible. If patients are not safe, whether to continue to let patients discharged with drainage tube? If patients are safe, is it worth advocating patients discharged with drainage? After all, discharging with drainage may significantly reduce the burden of the pancreatic center all over the country.

\section{Limitation}

The small sample size and the non-RCT study was our limitation. And according to the policy of the different country, is fasting for 2 weeks has more benefice to the cost-effectiveness, we don't know. More RCT studies are needed. 


\section{Conclusions}

To sum up, the new ERAS program implemented in this study was found to be feasible and safe for distal pancreatectomy surgery and leads to promising outcome. And no patient discharged with drainage in ERAS group. Such ERAS program, especially for DP, should be established.

\section{Acknowledgments}

Funding: This study was supported by grants from the Guangci Outstanding Youth Training Program (GCQN2017-B06).

\section{Footnote}

Conflicts of Interest: All authors have completed the ICMJE uniform disclosure form (available at http://dx.doi. org/10.21037/tcr.2019.10.26). The authors have no conflicts of interest to declare.

Ethical Statement: The authors are accountable for all aspects of the work in ensuring that questions related to the accuracy or integrity of any part of the work are appropriately investigated and resolved. The study was conducted in accordance with the Declaration of Helsinki (as revised in 2013). This study was approved by the ethics committee of Ruijin Hospital. And the study protocol was also approved by the institutional review board at Ruijin Hospital (ID of ethical approval: 2018-172). Individual consent for this retrospective analysis was waived.

Open Access Statement: This is an Open Access article distributed in accordance with the Creative Commons Attribution-NonCommercial-NoDerivs 4.0 International License (CC BY-NC-ND 4.0), which permits the noncommercial replication and distribution of the article with the strict proviso that no changes or edits are made and the original work is properly cited (including links to both the formal publication through the relevant DOI and the license). See: https://creativecommons.org/licenses/by-nc-nd/4.0/.

\section{References}

1. Gouma DJ, van Geenen RC, van Gulik TM, et al. Rates of complications and death after pancreaticoduodenectomy: risk factors and the impact of hospital volume. Ann Surg
2000;232:786-95.

2. Bassi C, Marchegiani G, Dervenis C, et al. The 2016 update of the International Study Group (ISGPS) definition and grading of postoperative pancreatic fistula: 11 Years After. Surgery 2017;161:584-91.

3. Besselink MG, van Rijssen LB, Bassi C, et al. Definition and classification of chyle leak after pancreatic operation: A consensus statement by the International Study Group on Pancreatic Surgery. Surgery 2017;161:365-72.

4. Wente $M N$, Bassi C, Dervenis C, et al. Delayed gastric emptying (DGE) after pancreatic surgery: a suggested definition by the International Study Group of Pancreatic Surgery (ISGPS). Surgery 2007;142:761-8.

5. Kehlet H. Multimodal approach to control postoperative pathophysiology and rehabilitation. Br J Anaesth 1997;78:606-17.

6. Nikfarjam M, Weinberg L, Low N, et al. A fast track recovery program significantly reduces hospital length of stay following uncomplicated pancreaticoduodenectomy. JOP 2013;14:63-70.

7. Abu Hilal M, Di Fabio F, Badran A, et al. Implementation of enhanced recovery programme after pancreatoduodenectomy: A single-centre UK pilot study. Pancreatology 2013;13:58-62.

8. Coolsen MME, Dam RMV, Chigharoe A, et al. Improving Outcome after Pancreaticoduodenectomy: Experiences with Implementing an Enhanced Recovery After Surgery (ERAS) Program. Dig Surg 2014;31:177-84.

9. Robertson N, Gallacher PJ, Peel N, et al. Implementation of an enhanced recovery programme following pancreaticoduodenectomy. HPB (Oxford) 2012;14:700-8.

10. Lassen K, Coolsen MME, Slim K, et al. Guidelines for Perioperative Care for Pancreaticoduodenectomy: Enhanced Recovery After Surgery (ERAS®) Society Recommendations. Clin Nutr 2012;31:817-30.

11. Deng X, Cheng X, Huo Z, et al. Modified protocol for enhanced recovery after surgery is beneficial for Chinese cancer patients undergoing pancreaticoduodenectomy. Oncotarget 2017;8:47841-8.

12. Bassi C, Molinari E, Malleo G, et al. Early versus late drain removal after standard pancreatic resections: results of a prospective randomized trial. Ann Surg 2010;252:207-14.

13. Lu X, Wang X, Fang Y, et al. Systematic Review and MetaAnalysis of Pancreatic Amylase Value on Postoperative Day 1 After Pancreatic Resection to Predict Postoperative Pancreatic Fistula. Medicine (Baltimore) 2016;95:e2569.

14. Lee CW, Pitt HA, Riall TS, et al. Low Drain Fluid 
Amylase Predicts Absence of Pancreatic Fistula Following Pancreatectomy. J Gastrointest Surg 2014;18:1902-10.

15. Wente MN, Veit JA, Bassi C, et al. Postpancreatectomy hemorrhage (PPH): an International Study Group of Pancreatic Surgery (ISGPS) definition. Surgery 2007;142:20-5.

16. Lillemoe KD, Kaushal S, Cameron JL, et al. Distal pancreatectomy: indications and outcomes in 235 patients. Ann Surg 1999;229:693-8; discussion 698-700.

17. Kleeff J, Diener MK, Z'Graggen K, et al. Distal pancreatectomy: risk factors for surgical failure in 302 consecutive cases. Ann Surg 2007;245:573-82.

18. Lee SY, Allen PJ, Sadot E, et al. Distal Pancreatectomy: A Single Institution's Experience in Open, Laparoscopic, and Robotic Approaches. J Am Coll Surg 2015;220:18-27.

19. Sauvanet A, Partensky C, Sastre B, et al. Medial pancreatectomy: a multi-institutional retrospective study of 53 patients by the French Pancreas Club. Surgery 2002;132:836-43.

20. Pratt W, Maithel S K, Vanounou T, et al. Postoperative

Cite this article as: Wang Y, Li J, Weng Y, Deng X, Jin J, Peng C. A new enhanced recovery after surgery pathway for left-sided pancreatic cancer patients after distal pancreatectomy. Transl Cancer Res 2019;8(7):2613-2620. doi: 10.21037/tcr.2019.10.26 pancreatic fistulas are not equivalent after proximal, distal, and central pancreatectomy. J Gastrointest Surg 2006;10:1264-78; discussion 1278-9.

21. Chang YR, Kang MJ, Kim H, et al. The natural course of pancreatic fistula and fluid collection after distal pancreatectomy: is drain insertion needed? Ann Surg Treat Res 2016;91:247-53.

22. Tjaden C, Hinz U, Hassenpflug M, et al. Fluid collection after distal pancreatectomy: a frequent finding. HPB (Oxford) 2016;18:35-40.

23. Tyberg A, Karia K, Gabr M, et al. Management of pancreatic fluid collections: A comprehensive review of the literature. World J Gastroenterol 2016;22:2256-70.

24. Sierzega M, Kulig P, Kolodziejczyk P, et al. Natural History of Intra-abdominal Fluid Collections Following Pancreatic Surgery. J Gastrointest Surg 2013;17:1406-13.

25. Čečka F, Jon B, Šubrt Z, et al. The effect of somatostatin and its analogs in the prevention of pancreatic fistula after elective pancreatic surgery. Eur Surg 2012;44:99-108. 